
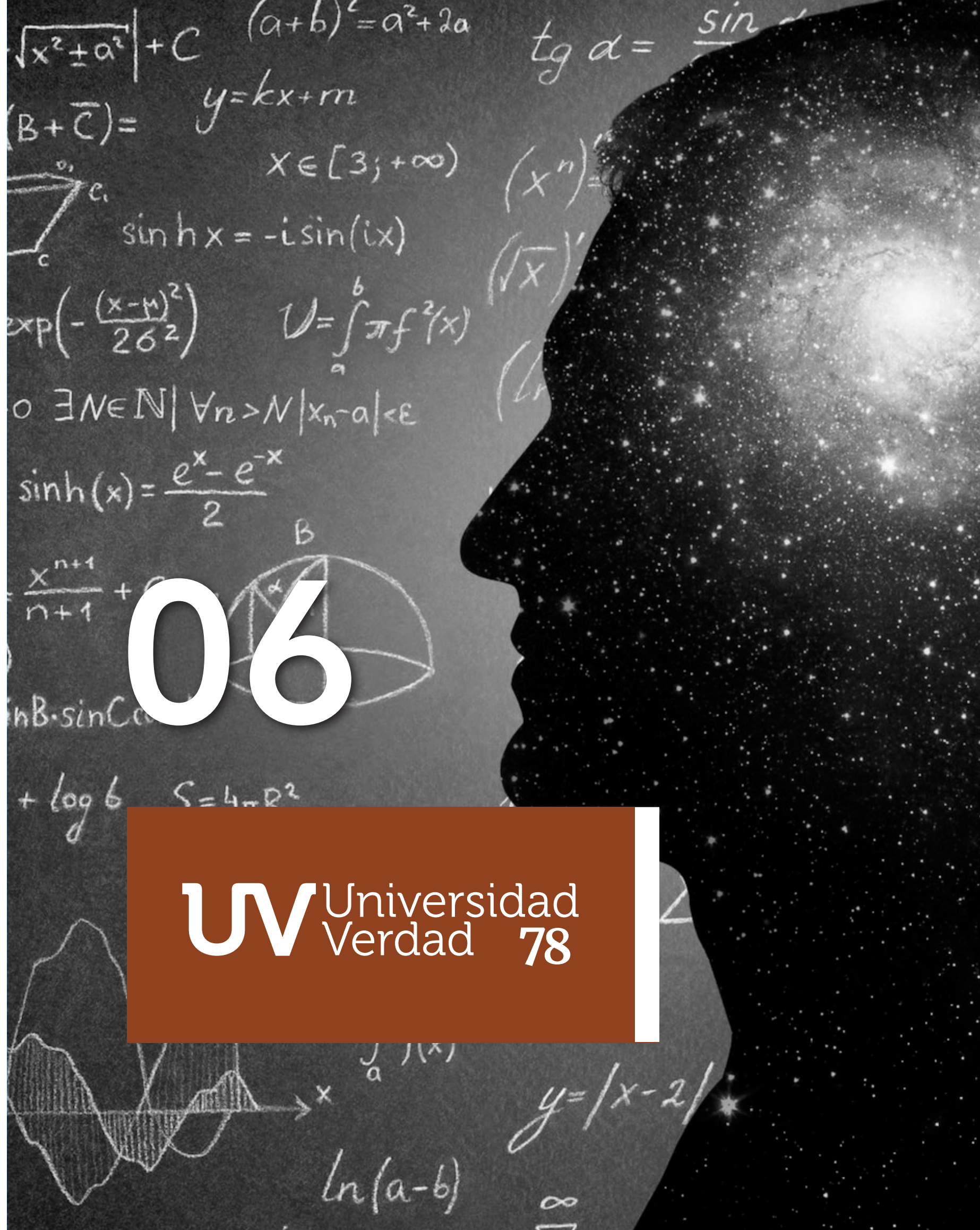

$\cos x=\operatorname{Re}\left\{e^{i x}\right\} \cos 20$ $x_{1}^{\prime}=1 \quad \sum_{k=0}$ 


\section{NECESIDADES, VALORES Y NORMAS DESDE UNA FILOSOFÍA CIENTÍFICA}

\section{Needs, Values and Norms from a Scientific Philosophy}

(iD Óscar Frederic Teixidó Durán, Escuela Técnica Superior de Ingeniería Agraria, Universidad de Lleida (España) (oscarburgo@hotmail.com)

(https://orcid.org/0000-0001-8557-0894)

\section{Resumen}

En este texto se intenta resumir la teoría axiológica y ética, así como su relación con los derechos humanos -DDHH- que se desarrolla desde la filosofía científica: una filosofía que busca construir sistemas conceptuales generales, que sean claros y exactos, y pretende ajustar tales sistemas a las ciencias de su tiempo, retroalimentarse con ellas. De esta forma, se indaga en la filosofía práctica encarada con el máximo rigor posible y centrada en lograr cierta objetividad; una filosofía técnica. A lo largo del trabajo se desarrollarán los conceptos, hipótesis y prescripciones centrales de la filosofía práctica sobre el sustrato científico disponible. También se proponen algunas ideas críticas con los 'derechos humanos' que pueden ser de interés para juristas y teóricos del derecho. El fin de tales críticas no es la refutación o supresión de los DDHH, sino todo lo contrario: su reafirmación contundente con las mejoras y reajustes que se necesiten.

\begin{abstract}
In this text we try to summarize the axiological and ethical theories, as well as its relation with human rights - HR- that is developed from scientific philosophy: a philosophy that seeks to construct general conceptual systems that are clear and exact, and tries to adjust itself to the sciences of its time, to feed back with them. In this way, it is investigated in the practical philosophy faced with the maximum possible rigor and focused on achieving certain objectivity; a technical philosophy. All along this work we develop the central concepts, hypothesis and prescriptions of the practical philosophy on the scientific substrate available. It also proposes some ideas critical of 'human rights' that may be of interest to jurists and legal theorists. The final objective of these critics it is not the refutation or suppression of HR, but just the opposed: their strength reaffirmation with the improvements and readjustments required.
\end{abstract}

\section{Palabras clave}

Valores, necesidades, normas, derechos, deberes, filosofía científica.

\section{Keywords}

Values, needs, norms, rights, duties, scientific philosophy. 
1.

\section{Introducción}

La filosofía práctica es la colección conceptual de todos aquellos campos de investigación filosófica que tratan sobre el obrar humano, dicho en términos generales. En la forma de filosofía, que se expondrá, tales campos que se suelen considerar son: la metodología, la axiología, la ética y la praxiología. Se explicarán tales áreas de estudio y sus relaciones objetivas de forma sistemática, hasta alcanzar a los archiconocidos derechos humanos (DDHH). Dado que las ciencias no se discutirán en los siguientes razonamientos y no se adentrará en exceso en sus diversas formas de proceder y teorizar, el desarrollo de la exposición se centrará aquí en la filosofía práctica más social y valorativa: la ética y la axiología así como la praxiología que se deriva de éstas. La metodología incumbe a diferentes valores y valoraciones. Sin embargo, no mantiene una ligadura tan estrecha como la axiología con la ética o la praxiología sobre la sociedad. Se la aludirá de todas formas, al tratar de normas, pero quedará en un segundo plano para centrar la reflexión en la sociedad per se; i. e. los sistemas sociales. Por otro lado, y como se podría pensar, la estética también tiene grandes compromisos axiológicos así como con la sociedad; pero al no ser una rama práctica "objetivable" (se asume, al menos en cuanto a su prescripción universal) sino de análisis y explicación al tratar valores subjetivos, como se dirá, queda relegada en esta exposición.

\subsection{Metodología}

Se guiará la explicación con apartados, introducidos por interrogantes, y encadenados entre sí sucesivamente; el marco de coordenadas desde el cual se encarará esta exposición de la filosofía práctica se realizará desde la filosofía científica.

Esto es, se adopta la metodología, que a continuación se justificará pertinentemente, de proponer tesis en forma de hipótesis claras que puedan evaluarse según su coherencia tanto propia como con los conocimientos teóricos de otras ramas de la filosofía, y de las ciencias contemporáneas. Se procederá por varias tácticas pues: ofreciendo ejemplos y contra-ejemplos, formulando sistemas teóricos que den respuesta a ciertas inquietudes de forma lógica y con ayuda de herramientas matemáticas, o bien buscando contradicciones en tesis contrarias por reducción al absurdo, etc. siguiendo la estructura mencionada de preguntas-respuestas provisionales y aumentando gradualmente el rigor con el que se trata el problema abierto en cada sección.

\subsection{La filosofía científica}

\section{¿Qué es la 'filosofía científica'?}

Los términos 'filosofía científica' son el nombre enfático con el que se alude a la filosofía realizada con rigor en sus teorías (entendida como una disciplina con ciertos límites), análogamente como sucede al hablar de 'medicina científica'. Esto es una filosofía que sea libre de ambigüedades, y sin quedar nunca apartada de la realidad. En relación y sinergia respecto a las diferentes ciencias contemporáneas. Y de la misma forma que en la medicina, con rigor (entendida como disciplina), únicamente existen técnica y tecnología modernas que se basen en la ciencia, siendo realmente redundante agregar más; de igual forma, de filosofía, con rigor, únicamente existe la que es clara e informada por la ciencia, sin agregarle más. Sin embargo, dado el contexto social presente, se cree necesario reivindicar su enlace con la claridad y las ciencias, igual que en medicina. Aunque solo sea un retoricismo para hablar de filosofía seria (filosofía, sin más). 
Tal propuesta metafilosófica (filosofía de la filosofía) asume un cientifismo tácito. La ciencia sería un modo privilegiado de conocer, al menos especializadamente, sobre el cual asentarse. Pero este no es un cientifismo duro o 'hardcore': no asume que la ciencia sea el único conocimiento, tampoco se la idealiza en "La Ciencia" (como un absoluto); todavía menos se la defiende dogmáticamente como infalible, o se la ideologiza como si fuese el único pilar que se deba preservar en la sociedad y en la cultura (Haack, 2017). Se trata de un cientifismo moderado o 'light' compatible con la filosofía, en igualdad (para que se puedan justificar una en la otra), y tolerable a otros conocimientos en otros órdenes cognoscitivos: como podrían ser la intuición, el conocimiento ordinario y la técnica simple, etc. Esto da lugar, pues, a un filosofismo ('light' de nuevo) implícito: la filosofía no es lo único que importa, pero es una manera también privilegiada para entender, al menos de forma general, sobre otras formas de conocimiento genérico como podrían ser los mitos o la religión.

Luego formalmente, si $F$ y $C$ son $n$-tuplas que representan la filosofía y las ciencias respectivamente, $\mathrm{y} \neq$ la desigualdad:

$$
\mathrm{F} \neq \mathrm{C}, \mathrm{F} \leftrightarrow \mathrm{C}
$$

Dónde $\mathrm{F} \leftrightarrow \mathrm{C}$ representa la relación de sinergia teórica entre ciencias y filosofía, en la que ' $\exists x \in \mathrm{C}: \tau \mathrm{F}(\mathrm{x})$ ' \& ' $\exists y \in \mathrm{F}: \tau \mathrm{C}(\mathrm{y})$ ' y los predicados $\tau \mathrm{F}$ y $\tau \mathrm{C}$ son los supuestos de trasfondo en filosofía y en ciencias también respectivamente, definido el trasfondo como un conjunto de ideas implícitas y explícitas presentes en cierta disciplina. Esto es, existe un elemento de la ciencia que es su trasfondo de supuestos filosóficos, y un elemento en la filosofía que es su trasfondo de supuestos científicos.

Tal relación vinculante entre ciencia y filosofía, que no es viciosa o cerrada sobre sí misma, si no virtuosa por ser constructiva; ya ha sido tanteada por muchos autores y sistemas filosóficos, pasados y también relativamente recientes como en el Integracionismo de Ferrater Mora (Velásquez, 2015). Se puede además considerar a la filosofía científica, como marco metafilosófico, un naturalismo epistemológico moderado (Diéguez, 2012) dado que se propone una continuidad entre filosofía y ciencia sin igualaciones; pero en estrecho contacto y con similitudes dentro de sus diferencias. Por lo tanto la metafilosofía asumida en la filosofía científica pone en estrecha relación a la filosofía con las ciencias punteras; pero de una forma razonable, que no pretende agotar toda forma de conocimiento y cognición. Ni siquiera tal forma de filosofía agota la diversidad de estudios filosóficos, puesto que, aun postulada la filosofía científica como la mejor forma de proceder cuando pueda hacerse (y a pesar de no ser original en su propuesta), admite grados de acercamiento y análisis preliminares varios: la protofilosofía -i. e. filosofía embrionaria pero en camino de tener rigor en su claridad y congruencia-, la semifilosofía -i. e. filosofía que ya puede ser rigurosa; pero aún dispone de áreas algo atrasadas-, etc. Es comprensible que, en tiempos de protociencia, solo podría existir en sinergia una protofilosofía dada; pero se tendría un mayor estatus cognoscitivo que otras formas de entendimiento todavía más borrosas y desligadas de proto-conocimientos especializados, por camino que quizás todavía le quedasen por recorrer a la filosofía embrionaria hasta su adultez.

Es decir, la filosofía científica busca ser dinámica, autocrítica y separarse también de "filosofías" especulativas, ofreciéndose como alternativa con ya larga tradición: una filosofía clara, sistémica, también exactificable con herramientas formales a veces, e informada siempre por las ciencias disponibles: ya sean formales (matemáticas, lógica) o fácticas (física, química, biología, psicología, sociología, etc.). Es por tanto una tarea clásica antes que nueva, con precursores como Aristóteles y la protociencia helénica, y más recientes como el científico L. Boltzmann (Romero, 2017) con el surgimiento de la ciencia moderna. Por otro lado evita caer en la exegesis y el mero 'comentarismo' de autores, optando por enfrentar problemas directos con hipótesis y sistemas teóricos -sistemas hipotético-deductivos-, a modo de respuestas.

El procedimiento de evaluación y revisión permanente de tales hipótesis y/o teorías propuestas es el testeo de su consistencia interna (coherencia lógica) y su coherencia externa (coherencia con otros campos de conocimiento, tanto filosóficos también, como científicos), así como por la fertilidad (ayuda, guía y facilitación) a la que contribuya en los avances del entendimiento y el cambio social; siendo 
este el requisito que permite decidir entre teorías igual de consistentes y congruentes con la ciencia. Los problemas y formas de estudio filosófico informado por las ciencias son diversos, pero pueden agruparse aún en las ramas clásicas de la filosofía: lógica (teoría de la inferencia), semántica (teoría del significado), ontología (teoría de las entidades más generales del mundo), gnoseología (teoría de las formas de obtención del conocimiento), metodología (teoría de los modos de indagación), axiología (teoría de los valores), ética (teoría de las normas de conducta), praxiología (teoría sobre la acción humana) y estética (teoría de los valores de la belleza). Siendo la metodología, la axiología, la ética y la praxiología las ramas prácticas o la filosofía práctica. No cabe confundir, pese a ser muy habitual, la filosofía de la ciencia, capítulo de la gnoseología (a su vez epistemología si trata sobre ciencias), con la filosofía científica. Es desde esta última posición que se partirá para exponer la filosofía práctica. Y es que saber de normas en sociedad; ética, implica explicar fines y valores, juicios de valor, etc. Y para hablar de valores y juicios de estos, axiología, cabe hablar de sus raíces. Si se busca una raíz objetiva para la posibilidad de unos valores objetivos, cabe entonces hablar de unas raíces del valor que también sean objetivas, i. e. precisas y libres de sesgos evidentes. Lo cual implica que son pasibles a ser investigadas científicamente. Quizás la raíz buscada de los valores no sea una sola, si no varias raíces: las necesidades de los seres vivos.

\section{2.}

\section{Necesidades}

\section{¿Qué es una necesidad?}

Una necesidad se suele entender como un requerimiento, algo que cabe cumplir en algún aspecto de algún tipo, por parte de un ser vivo: $A$ requiere $B$ para $C$. Pero es evidente que un ser vivo requiere de algo en ciertos momentos que en otros momentos no, como cuando un ser humano come en función de la hora que sea. También es evidente que un ser vivo requiere de algo en algunos lugares y que en otros no, como cuando cualquier animal sufre de sed en el desierto. Depende de sus medios y disposición. Por lo tanto, una necesidad es un requerimiento, que depende de un ser vivo y sus circunstancias - lo cual puede recordar a la famosa frase de Ortega y Gasset (1914)-. El hecho de que dependa de algún ser y de su contexto hace que las posibles raíces del valor sean relativas, más no necesariamente subjetivas, al menos por lo deducible hasta ahora. Sin embargo, también existen muchos tipos de requerimientos circunstanciales. Si un ser vivo animal (lo suficientemente complejo), desea jugar con algún objeto o cuerpo, posiblemente puede dejar de jugar con ello en cualquier momento, sea arbitraria o deliberadamente. Parece lo primero algo relativo y además subjetivo. En cambio, está claro que el hambre no se agota arbitraria o deliberadamente. Esto segundo es objetivo (no-sesgado), aún relativo a especie y circunstancias, lo cual implicará también su debido trato razonable (Rescher, 1999) si se busca preservarlos.

Se puede discernir, pues, entre requerimientos de necesidad y deseo. Y si estos dos tipos de requerimientos serán lo que fundan ${ }^{1}$ los valores y a las valoraciones, y se busca exponer sobre lo que es objetivo, habrá que tratar de necesidades antes que de deseos. También hay otros tipos de requerimientos: no es lo mismo requerir de atención que requerir de un riñón o paz social. Hay requerimientos biológicos, psicológicos y sociales. Luego hay necesidades y deseos psicológicos, biológicos y sociales; a veces intersecados. Y también hay requerimientos reales o necesidades, más o menos urgentes: no es lo mismo enfrentar una guerra que fomentar la información óptima entre comunidades sociales; no es lo mismo comer para sobrevivir que mantenerse sano; no es lo mismo padecer de una enfermedad mental que entrenar una facultad cognitiva. Las necesidades, y sólo éstas, pueden ser de supervivencia (i. e. seguridad) o de salud (i. e. sanidad).

1. Se diferencia entre 'fundamentar' y 'fundar': en el texto se usará sobre todo la primera expresión, como un razonamiento lógico desde una base unívoca, piramidalmente (y cuya idea se rechazará). La segunda se usa aquí como sinónimo de justificar, de usar ideas, conceptos, cosas y etc., de "manera fundada". 


\subsection{Necesidades como déficits reales}

\section{¿Cómo definir con rigor una necesidad?}

Con lo anterior, se puede definir una necesidad como un requerimiento real y circunstancial de un ser vivo; ergo una función de estado de un organismo vivo, pues cambia dependiendo del estado del ser vivo en cuestión. A veces es cuantificable, y entonces se habla de un déficit de un organismo. Dados los tipos de déficit que hay como funciones de estado, también es posible decir que los hay biológicos, psicológicos y sociales (dados organismos con aspectos no sólo biológicos, si no también biopsicológicos, sociales y hasta culturales). Estos dos últimos solo por parte de animales gregarios. Por lo tanto, las necesidades se pueden considerar déficit biopsicosociales que dependen de cada especie de seres vivos. De necesidades pues, los requerimientos reales y materiales, las disponen desde los microorganismos simples a los mamíferos complejos pasando por las esponjas marinas, las plantas y los insectos. Aunque como se puede apreciar y ya se ha señalado, no todos los animales comparten las mismas necesidades ni en número ni en cualidad, tampoco en modo (una colonia de bacterias, por numerosa que sea, stricto sensu no tiene ninguna necesidad social).

Ontológicamente (desde la disciplina filosófica que trata todos los existentes en general), se puede decir que las necesidades, como los deseos, son propiedades disposicionales de seres vivos en sus requerimientos. Son disposiciones de seres vivos en ciertos aspectos con cierta variedad de cosas; sean objetos, procesos. Pueden estudiarse por las ciencias naturales y sociales. Los valores se erigirían sobre tales necesidades y deseos, y serían los valores ligados a necesidades los que podrían ser objetivos.

\section{3.}

\section{Valores objetivos}

\section{¿Qué es un valor y en dónde radica su objetividad?}

La disciplina que estudia los valores es la axiología (Frondizi, 1958), la teoría de los valores. Se suele pensar ordinariamente que se dispone de un valor si se aprecia algo. Es decir, si se evalúa algo: superficialmente, se buscaría la generación de algo si se careciese de ese algo. Algunos seres humanos aprecian cosas como la verdad, la justicia, la belleza, el amor, etc. Y desprecian otras (disvalores) como la mentira, la injustica, la fealdad, el odio, etc. Un valor relaciona, y por lo tanto también tiene relatividad, pero no es igual a una necesidad.

El valor es una conceptualización. Por ello un valor se puede considerar un artefacto conceptual, aunque a veces también se lo propone como una cosa material y otras como otra propiedad relacional. Un valor, en todo caso, trazaría una evaluación de satisfacción o no, de requerimientos a cumplir y evitar discumplir: en necesidades o en deseos. En el caso de valores objetivos, su raíz sería, como se ha dicho, la necesidad biopsicosocial; fuera esta de salud o de supervivencia. Por ende también habría valores biopsicosociales objetivos o subjetivos. También valores biopsicosociales objetivos de salud o de supervivencia (valores básicos). Pero no siempre es posible cumplir todos los valores. Hay conflictos entre ellos y entre los diferentes niveles. Se puede hablar de una dialéctica (contradicción, choque conceptual) y una ponderación sobre tal conflicto, una decisión ulterior entre los valores en juego. Por lo tanto, de tal dialéctica emergen juicios de valor; las valoraciones. A veces a éstas también se las considera ajenas al sujeto y al contexto, otras veces se las hace muy dependientes de cada individuo. 
3.1. Valores objetivos como ficciones sobre déficit en sistemas de valor

\section{¿Cómo ofrecer las nociones precisas del valor obje- tivo, y de las escalas i. e. sistemas objetivos de valor?}

Los valores per se pueden entenderse mejor como artefactos conceptuales, ficciones, más que como materia o propiedades reales en sí mismos (Teixidó, 2019, 2020). De por sí no se ubican en el espacio-tiempo, no cambian y no tienen $E$ energía asociada a $x$ valores como $E(x)$, ni una diferencia de entropía $d S$ asociada a $s$ sistema de valores como $d S(s)$. Tampoco pueden ser propiedades, puesto que sería redundante considerando ya el rol de las necesidades, y de considerarse los valores como tales, entonces se tratarían de procesos neurobiológicos y conductas asociadas con seres, cosas y circunstancias, pero no a los valores per se, tal cual se los tratan en valoraciones y juicios por parte de los seres vivos que los idean. Son ficciones, no cosas ni rasgos o propiedades de cosas. Se inventan o construyen sobre ciertos requerimientos. Esto no tiene porqué llevar al idealismo de los valores: aun siendo ficciones los valores no tienen existencia autónoma o reificada. Su existencia puede ser fingida y proponerse solo conceptualmente, de modo que podría ser compatible con un materialismo pluralista, y emergentista (Ferrater, 1999). Por ello también podemos decir que, mientras todos los seres vivos tienen unas necesidades, no todos tienen deseos, y todavía menos disponen de valores o de capacidad para inventarlos. En concreto sólo podrían valorar los animales más complejos. Ontológicamente el valor tendría inicio de su existencia, que se sepa hoy, gracias a la biología evolutiva, la filogenia, la paleontología y la geología, hace unos cuatrocientos millones de años aproximadamente (esto no implica que se dieran ya, sino que su posibilidad era factible alrededor de aquellas fechas holgadas, se entiende).

Además de la dialéctica entre valores se puede decir que surgen los juicios de valor, elecciones de prioridad, y por lo tanto la valoración. Que al ser relativa y sobre ficciones conceptuales, sería dependiente siempre de los animales que valoren también. Pero de nuevo, no agotaría su objetividad por ello. Como artefactos conceptuales inventados que evalúan requerimientos, es factible definir los valores formal- mente (Romero, 2018) sea con [1] lógica relacional mediante o empleando [2] $n$-tuplas. Esto es:

$$
\begin{aligned}
& \text { Vabcd... } \\
& V(a, b, c, d, u)=V
\end{aligned}
$$

En donde: $V$ designaría al valor, a al sujeto que valo$\mathrm{ra}, \mathrm{b}$ al aspecto valorado del sujeto, c a aquella cosa (objeto, rasgo, proceso; "el objeto de valor") que satisface el aspecto valorado del sujeto, y d las circunstancias de valoración del sujeto de algo en un aspecto. En el caso de [1] se pueden encadenar más y más variables. En [2] con las variables ya dadas en la 5-tupla o péntupla, se puede disponer de una cuantificación del valor en un intervalo $u$ (generalmente una graduación continua $[-1,+1]$ ) que da lugar tras su evaluación, a cierta cantidad $v$. No siempre es viable cuantificar valores; eso sólo estaría reservado para valores objetivos. Y sólo algunos de ellos podrían ser bien investigados científica y tecnológicamente; pero en todo caso es a priori posible. Y también pues, el conocimiento de las diversas construcciones de valor es siempre provisional; revisable. Los valores (Bunge, 1989) podrían ser objetivos 0 (p.ej.: la cohesión social), o subjetivos $S$ (p.ej.: la gula) o bien ambos a la vez, $0-S$ (p.ej.: la felicidad o placer hacia la cohesión social percibida, y la cohesión social misma apreciada). Como se dijo; los valores sobre necesidades forman valores objetivos, y sobre deseos o intereses, valores subjetivos; pero también pueden todos estos biovalores, psicovalores o sociovalores, ser subjetivos u objetivos. Y cada valor biopsicosocial objetivo puede ser o de supervivencia o de salud (Tabla 1). Todo este tratamiento sistémico de los valores puede sintetizarse en: 


\section{Tabla 1}

Tipos de valores objetivos según necesidades y clase

\begin{tabular}{|l|l|l|l|}
\hline $\begin{array}{c}\text { Necesidades/ } \\
\text { Valores }\end{array}$ & \multicolumn{1}{|c|}{ Biológicos } & \multicolumn{1}{c|}{ Psicológicos } & \multicolumn{1}{c|}{ Sociales } \\
\hline Supervivencia & Biovalores de supervivencia & Psicovalores de supervivencia & Sociovalores de supervivencia \\
\hline Salud & Biovalores de salud & Psicovalores de salud & Sociovalores de salud \\
\hline
\end{tabular}

Los valores, como ficciones evaluativas diversas, pueden organizarse en sistemas relativos a cada ser vivo. Y más objetivamente, relativos según la especie; pero dentro de ésta, aproximadamente válidos para cada ser vivo en sus déficits reales; todavía dependiendo de circunstancias como nivel de desarrollo, medios disponibles, etc. En general, todo sistema o escala de valores cualquiera $(x)$ equivocada o no, razonable o no, subjetiva o no, establece grados de prioridad de valores en orden de $\mathrm{N}$-ariedad. Es decir, poniendo como ejemplo tres niveles de valor en conflicto con > priorizaciones:

\section{(x) VP > VS > VT}

Donde VP es el valor primario, VS el valor secundario y VT el valor terciario. Está claro que racionalmente, entre conflictos dialécticos entre VP-VS, en juicios de valor razonables se priorizaría VP y entre VP-VT, VP también. Por lo tanto, deductivamente, entre VS-VT, prioriza VS. Todo aquel objeto de valor o valioso en alguna de tales categorías, i. e. que satisface VP, VS \& VT en algún aspecto, se puede considerar un 'bien'. Quedan entonces definidos los 'bienes' y sus disvalores, los 'males' (que no unos valores idealizados como "El Bien" /"El Mal" que puedan reificarse o hipostasiarse, desligarse de los sujetos pensantes). Pero estas escalas siempre se pueden organizar de diferentes formas, con muy distintos niveles, aspectos distintos inconmensurables y con mayor o menor racionalidad en su planteamiento y cumplimiento. De igual forma que no es fácil clasificar los objetos de valor, cuantificar valores u ordenarlos de forma absoluta, es difícil hacer lo mismo con los sistemas de valor, incluso tomando sistemas por separado frente a los demás. Hay sistemas de valores estéticos, artísticos, morales, cognitivos, lógicos, inconsistentes, fraternales, apáticos, etc.

En general y para simplificar, se propondrán dos tipos de valores esenciales que pueden llegar a enfrentarse, y su debida priorización: se asociarán los valores objetivos $O$ con los valores primarios $(O=V P)$ biopsicosociales (con los de salud y supervivencia como básicos dentro de VP a su vez, aunque es una simplificación y podría decirse que la supervivencia prima sobre la salud, pese a que no necesariamente sean incompatibles ambas) y los valores subjetivos biopsicosociales $S$ con lo secundario $(S=V S)$. Lo primario reside en los valores sobre necesidades (valores básicos, objetivos) y lo secundario en aquellos valores sobre deseos. En tal micro-sistema axiológico (A) pues;

\section{(A) VP > VS}

Por ejemplo, la compra de vehículos bellos y rápidos siempre sería correcta a menos que de alguna forma impidiera conseguir un mínimo de alimentos nutritivos para alguien; entonces priorizaría los alimentos sobre la compra de vehículos. Esta escala se violaría, de hacerse lo contrario, y sería alterna a escalas donde se asociara lo subjetivo con lo primario y lo objetivo con lo secundario. Por ejemplo, para un asesino quizá matar por gusto estético sea priorizable frente a salvar y mantener vidas (esto es: $S=V P, O=V S$ ).

Aquí se asume, como primera norma de un sistema axiológico razonable y objetivo: compatibilizar felicidad y bienestar siempre que se pueda, pero priorizar siempre ante todo conflicto valorativo el bienestar biopsicosocial de supervivencia y salud. 
4.

\section{Normas}

\section{¿Qué es una norma (social)?}

Generalmente cuando se habla de normas o reglas se entiende que éstas son pautas o enunciados instructivos sobre qué se debe hacer para lograr algo, no de lo que es el caso. Y ese algo es una cosa apreciable, valorable. En una sociedad, esto implicará pautas de conducta en sistemas sociales que deben reglarse para alcanzar unos valores. De esta forma, se postula que toda norma está ligada a valores, y que toda norma va encaminada a poder cumplir con uno o más valores, sea de forma mediata o inmediata, y sea imperando o inhibiendo una conducta: regulando el comportamiento.

La justificación de qué normas son correctas y cuáles no siempre ha estado cargada de polémica. Anteriormente se expuso una norma axiológica: para lograr bienestar real, proteger los valores objetivos i. e. primarios en cualquier dialéctica con otros valores. Sin embargo, también hay escalas axiológicas de mínimos que pueden invertir el orden de prioridad, como bien podría hacerlo un asesino (como antes se ejemplificaba). No parece a priori que exista una forma racional y absoluta de establecer qué escala o sistema axiológico es mejor que otro en la teoría o en la práctica. Esto hace que la objetividad, pese a darse en valores y normas, no parezca ser importante para una axiología prescriptiva. Es decir, si un valor 0 y su norma asociada es objetivo o subjetivo, no permite deducir su mayor prioridad necesaria a riesgo de cometer falacias, pudiéndose éstas englobar en una falacia de justificación cognitiva (Zavadivker, 2004). Sobre las distintas falacias que encierra tal encadenamiento lógico, se ha expuesto bastante con anterioridad (Moore, 1922): una falacia ser-deber ser o Guillotina de Hume se comete al identificar la prioridad con lo objetivo por ser algo fáctico e investigable científicamente; una falacia naturalista como expuso Moore, si se identifica el 'bien' con cualquier aspecto parcial, pero dado por esencial del 'bien' (aunque Moore iba tan lejos que rechazaba cualquier definición objetiva). La falacia de justificación cognitiva reuniría todos los intentos fallidos de fundamentación unívoca, siendo solamente irrelevancias.

Sin embargo, incluso aceptando que es totalmente imposible derivar el valor y las normas sin más, del hecho, que es imposible una fundamentación última de cualquier tipo en cualquier sistema de valores, $\mathrm{y}$ que el bien suele definirse parcialmente; nada de esto invalida que pueda trazarse lo postulado de manera fundada, y además hacerse sin arbitrariedad, como anteriormente se expuso. Al fin y al cabo:

(i) Se asocia un carácter primario en valores y normas a la objetividad de los valores y normas, no a los hechos mismos barajados por estos;

(ii) Los valores y los 'bienes' se pueden tratar objetivamente aún sin fundamentación. Y una axiología sin fundamentos no implica necesariamente una axiología subjetiva, de igual forma que una axiología relativa tampoco lo implica;

(iii) De todas las escalas de valor, solo una de los virtual e igualmente arbitrarios infinitos sistemas axiológicos posibles, ayudaría a responder adecuada y racionalmente a las necesidades. Mientras que todos los demás no, o bien lo harían menos. Y de ser el propio sistema una de las escalas de valores que menos ayudarían, siempre se tendría ya la posibilidad de que tal sistema axiológico más objetivo y razonable existiera.

Adicionalmente, pero derivado de lo anterior: (iv) realmente es muy dudoso que ninguna disciplina en general, sea teórica, práctica o mixta, tenga de fundamentos últimos (todo sistema teórico es hipotético-deductivo; provisional y revisable sin un principio o un final absolutos). Y se repite, eso no hace a todas las ciencias y filosofías inviables.

Por ende, aún sin inferir necesidad en su defensa, y asumiendo así que un ser racional pueda optar por otras escalas de valor arbitrarias; se puede afirmar que la escala (A) es al menos más objetiva, y no arbitraria, y que normas asociadas al sistema axiológico (A) son posibles (faltaría ver si a parte de posibles, unas u otras reglas son más o menos efectivas en sus propósitos valorados). Hay una asimetría existente 
entre (A) y los sistemas axiológicos $\neg(A)$ por cuanto, aun igual de razonables todos para con los fines de cada cual, sólo unos pocos sistemas pueden plantearse objetivamente y evaluarse según si satisfacen mejor o peor las necesidades humanas (y también las animales no-humanas). Esto es suficiente para marcar una diferencia real. De igual forma que en una ingeniería se podría empezar a estudiar cómo hacer menos eficientes unos procesos industriales, para lo cual también existen infinitas formas de lograr tal fin, pero sólo un número bajo de construir artefactos que se conserven y optimicen resultados.

Por lo que las anteriores falacias se llegan a aceptar y pueden evitarse de tal modo, incluso asumiendo una concepción más teórica y provisional de la axiología teórica y práctica. Una norma puede deducirse de otras normas, y junto a un valor explícito en un sistema axiológico, puede llegar a inferirse de hechos aún sin nunca poderse reducir a éstos (y la inversa, la falacia moralista, tampoco: no se pueden deducir hechos de deberes o normas, o reducirlas a estos). Y siempre teniendo en cuenta que lo razonable en normas y axiologías puede ser algo subjetivo u objetivo, pero que solo se considera aquí lo objetivo pese a que esto no sea fundamentado, porque, incluso, así marca una diferencia real con los demás sistemas no fundamentados y además arbitrarios (más subjetivos, por razonables o coherentes o exactos que lleguen a ser).

Por lo tanto, queda vía libre para formular sistemas de valores y también de normas, en las ciencias y en la sociedad misma tratada desde la filosofía. Esto da lugar a la práctica teórica, lo técnico; en lo cual la filosofía práctica conformaría un capítulo y devendría en una filosofía técnica, y con ello lo moral y ético también.

\subsection{Normas como reglas de conducta social}

\section{¿Qué es una norma (social) de forma precisa, que entronque con lo ético?}

Con todo lo anterior, queda claro que se puede hablar de normas que, aun dependientes de valores, mantengan contacto con los hechos (a los que son siempre irreductibles; i. e. no-deducibles en el plano teórico, aunque se usen para el razonar práctico).
Tales hechos pueden ser disposiciones materiales de déficit o intereses/deseos, y a los que atar unos valores que cabe cumplir mediante esas normas.

Una norma o regla sería pues una prescripción: la orden condicional de que, para lograr algo, cabe hacer o no hacer otra cosa. Y esto puede hacer una conversión a los hechos, a lo fáctico, pero no de la forma simple y falaz que suele mostrarse usualmente.

La lógica que subyace a las normas i. e. reglas, en general, puede establecerse como sigue a continuación (Bunge, 2012):

(a) Establecer un enunciado de hecho condicional (a poder ser, legaliforme, de ley).

(b) Darle un sentido pragmático al enunciado (de aplicación humana).

(c) Hacer una conversión del enunciado legaliforme a una pauta condicional de obrar (que podrá ser, bien negativa o positiva).

(d) Dado un valor, escoger cuál de las dos conversiones es la conveniente como regla.

Esto es, en donde $\rightarrow$ indica la implicación lógica condicional, $\neg$ designa la negación lógica y per el condicional práctico ("hacer algo para lograr otro algo"), luego;

(a) "Si en una sociedad hay distribución igualitaria de los recursos hídricos, los seres humanos se hidratan y sobreviven" o: $A \rightarrow B$

(b) "Hacer que una sociedad distribuya igualitariamente sus recursos hídricos, provoca que los seres humanos se hidraten y sobrevivan" o: $A \rightarrow B$ (no hay cambio lógico aún).

(c.1) "Si se busca que los seres humanos se hidraten y sobrevivan, hacer que una sociedad distribuya igualitariamente sus recursos hídricos" o: B per A

(c.2) "Si se busca que los seres humanos no se hidraten y sobrevivan, hacer que una sociedad no distribuya igualitariamente sus recursos hídricos" o: $\neg B$ per $\neg A$

(d) [Se asume aquí un biovalor $O$ de defensa de las necesidades humanas de nutrición] "Si se busca que los seres humanos se hidraten y sobrevivan, hacer que una sociedad distribuya igualitariamente sus recursos hídricos" o: B per A 
O simplemente: A. Donde la consecuencia práctica A para obrar se tomaría ya sin condicional, un puramente imperativo u obligatorio pues: "hacer que una sociedad distribuya igualitariamente sus recursos hídricos". También esto puede revocarse a una permisión si se niega la obligatoriedad, algo para lo cual la lógica modal puede ayudar.

Cómo se ve, se ha pasado lógicamente de (a) a (d). De hechos a normas, pero agregando una práctica necesaria y un valor. Por eso, como ya se dijo, dado que la falacia ser-deber ser consiste en deducir sin más normas de hechos, y aquí sólo se ha podido hacer teniendo en cuenta una praxis y un fin valorado: no se incurre en la misma. No se incurre en una falacia naturalista tampoco puesto que los 'bienes' se han definido en aspectos tanto objetivos como subjetivos, en categorías diferentes, y relativos a cada sistema o escala de valores, en su debido aspecto y contexto que sea relevante. Y tampoco se cae en una falacia de justificación cognitiva: el valor tomado no es algo deductivo y algorítmico a lo que todos deban llegar, como un fundamento unívoco, y por el simple hecho de basarse en hechos objetivos, las necesidades (aunque ésta es la única opción más racional y no-arbitraria). Más bien, como todo conocimiento, es siempre tentativo, sólo condicional y razonable como otras opciones, aunque en este caso las demás son más arbitrarias realmente al no basarse -que no fundamentarse - en requerimientos objetivos y materiales (hay una asimetría, la diferencia antes citada).

Pues bien, todo obrar tratado teóricamente (los planos dispuestos antes de construir) asume tales reglas, prescripciones o normas. La técnica, sea con artefactos tecnológicos o con instrucciones de pautas de conducta simple, puede analizarse de este modo. La filosofía práctica, por lo tanto, puede entenderse como filosofía técnica. Y la ética entonces, como una técnica filosófica: la rama filosófica que se encarga de explicar y de evaluar qué sistemas morales (de normas de conducta general en sociedad) son mejores que otros para alcanzar el 'bien'. Meta-éticamente, estaría en gran y estrecho contacto con la axiología, discurriendo en paralelo con la praxiología para conocer las mejores formas óptimas de obrar con eficacia e inferencia práctica, y también discurriendo en paralelo a la estética, aunque esta sin poder prescriptivo no-arbitrario. Los 'bienes', como se ha dicho antes, de conectarse a la axiología, pueden ser de distintas formas pero siendo siempre los más prioritarios los primarios en el sistema: el bienestar objetivo (valores biopsicosociales básicos de salud y seguridad).

La ética como sistema, por un lado, como la axiología, describiría y explicaría (y usaría tal explicación para luego aplicar medidas) los sentimientos morales, el devenir histórico de diferentes éticas y morales, etc. Por otro lado, la ética, como la axiología prescribiría -sería normativa respecto a - cómo comparar y escoger el mejor sistema o sistemas de normas en función del bienestar alcanzado (principalmente, humano). Esta es la diferencia entre ética explicativa y ética normativa. Los sistemas de normas de conducta social general que se deben evaluar serían los sistemas morales. Tales sistemas de normas pueden ser: subjetivos (pautar lo que le gusta a una sociedad), objetivos (pautar lo que una sociedad promueve sobre sus conocimientos) o mixtos; primitivos (proto-morales de sistemas gregarios animales) o sofisticados (sistemas complejos de leyes morales y de derecho); más o menos eficaces (una moral que logra poco bienestar comparado con otra que sí logra un alto índice); particularistas (normas locales o nacionalistas) o universalista (sistemas morales humanistas, o incluso animalistas).

La ética, además, en su práctica y aplicación directa se puede dividir en al menos tres campos especializados actuales de elevada prioridad - pero esta no es una clasificación exhaustiva-: (1) la nomoética o ética de las leyes, (2) la bioética o ética de las tecnologías biológicas, y (3) la tecnoética o ética de las tecnologías. Algunas de estas subdisciplinas intersecan, como (2) puede comprenderse dentro de (3). Y pueden darse más especializaciones de la ética con el paso del tiempo y futuras indagaciones.

En el cómo se contrastarían de facto todos los diferentes aspectos relevantes de estudio, que luego ya evaluará la ética más normativa y técnica para discernir entre mejores morales y peores morales; esto sería recurriendo indirectamente desde la filosofía a datos de las ciencias sociales y biosociales. Datos científicos y tecnológicos que traten hechos y tendencias estadísticas bajo las definiciones comunes y objetivas de los déficits antes expuestos (a riesgo de caer en encuestas de opinión fenoménica de los 
civiles). Estos datos deberían provenir de diferentes disciplinas: de la fisiología, la epidemiología, la neurociencia, la psicología, la psicología clínica, la medicina, la nutrición, la sociología normativa, etc. Establecer causalidades que puedan probar a ciertos valores y leyes morales como garantes de un bienestar de índice mayor o superior a otros códigos morales, es harto difícil; es una limitación cognitiva, teórica y tecnológica, que cabe reconocer, más no imposible, aún muy provisionalmente. Por otro lado, si tal trabajo con base empírica se considera filosofía experimental, que así sea.

Clásicamente existen muchos tipos de sistemas éticos y morales. Al tratar sobre la historia de la ética se hace de nuevo, meta-ética. Los más famosos sistemas éticos y algunas de sus morales, son tres: la ética de las virtudes o de la eudaimonia, de Aristóteles; la ética formal o deontologista, de Kant; y la ética consecuencialista-marginalista o utilitarismo, de Bentham y Mill. Todavía hay grandes defensores de todas estas tradiciones, si bien algunas tendencias quizá son más favorables a unas que a otras. Modernamente se han propuesto algunos nuevos sistemas éticos y morales. Los hay que prestan más atención a aspectos sociales concretos, como los políticos y legales, así como tratan de erigirse sobre anteriores tradiciones como el deontologismo y el contractualismo (Rawls, 2006). También en las últimas décadas se han postulado códigos endomorales (de y para la propia comunidad afectada) sobre los valores y normas de las ciencias (Merton, 1972), cosa que nutre a la metodología como rama también práctica y a fortiori técnica de la filosofía. Todos los sistemas normativos, como no podría ser de otra manera, tienen sus aciertos y errores, y como toda tecnología moderna clara y científicamente informada, limitaciones.

Dadas las diferentes versiones, teorías y tradiciones, una ética técnica clara e informada científicamente, deberá evaluar con rigor e igual a como evaluarán también aquellos sistemas clásicos más cercanos a la teorización técnica y científica, y priorizar así los sistemas morales más claros, efectivos y sistémicos. La evaluación ética técnica se haría así: al recabar datos del mundo deberá ser consecuencialista, pero también realista, no fenoménica (como lo es todo aquello centrado en lo experiencial: felicidad, placer, etc.), evaluando en cambio el bienestar real satisfecho, como prioritario, incluso teniendo en consi- deración la vivencia y la experiencia. Y al pretender leyes que todos puedan usar, cabrá partir de cierto universalismo, evaluando la fecundidad en el cambio social. Con estas premisas podrían evaluarse sistemas morales sobre el bienestar logrado: se estaría entrando, así, en la parte práctica o normativa de la ética, en contacto con las distintas morales. Pero no se adentrará ahora en discernir cuál de los anteriores sistemas éticos es más técnico y cercano a la ciencia o cuál de sus morales es mejor o si existe alguno ya óptimo: bastará aquí para proseguir y acabar, con asumir unos mínimos (Bunge, 2002).

Estos mínimos son proponer que las mejores formas de conducta hoy en día, al menos, pasan por considerar los derechos (en un sentido moral, general, de permisividad teórica) y los deberes (en un sentido moral, general, de obligación teórica) entrelazados entre sí, en proporciones 1:1, y buscando bienestar propio y ajeno, a la par. Esto es, todo deber implica un derecho y todo derecho implica un deber. En estos mínimos de equidad se toman los bienes individuales y los bienes ajenos en imbricación mutua y en general lineal, aunque puede tratarse en sistema y se deja abierto a excepciones o ampliaciones. Se puede decir, entendidos como predicados que, si D es un derecho y $\mathrm{R}$ un deber o responsabilidad -aquí se tomarán cómo sinónimos ambos términos - sobre $\mathrm{x}$ evento moral o de conducta social general relevante, $\mathrm{y} \Leftrightarrow$ designa la co-implicación (pero sin ser la equivalencia formal), entonces:

$$
\mathrm{Dx} \Leftrightarrow \mathrm{Rx}
$$

Por lo que, puesto en una prescripción más formal: (Dx $\Leftrightarrow \mathrm{Rx}$ ) per VP. O bien: "Hacer que derechos y deberes vayan parejos para satisfacer valores primarios". Tales pares derecho-deber y deber-derecho estarían conectados entonces a VP o VS, siendo o normas primarias o secundarias, dependiendo del evento x sopesado y el tipo de valores que estén en juego, siguiendo lo expuesto sobre axiología y sistemas normativos de conducta social general, moral. Y siendo siempre prioritario lo primario, en conflictos. Huelga decir que la demostración de tal postulado de co-implicación entre normas de derecho y deber no es auto-evidente (nada lo es en ciencias, filosofía o tecnología). Pueden existir varios modos de deducción del postulado a través de axiomas y reglas de inferencia, algunas compatibles entre sí. Pero no 
se adentrará ahora en ulteriores discusiones éticas. Basta con esta micro-teoría ética y moral para proseguir con el estudio sintético de una filosofía práctica que esté científicamente informada.

$\mathrm{Si}$, a veces, cabe priorizar derecho a deber o viceversa en dilemas morales o situaciones especiales alternas; si los derechos y deberes deben ser pasivos o activos i. e. negativos o positivos, o ambos; si se debe añadir una máxima moral de justicia o una pluralidad de máximas; si hacen falta reglas adicionales de conducta general; si cabe agregar consideraciones sobre las personas o sujetos morales; si se deben basar derechos y deberes en ideas de libertad, igualdad, fraternidad y equidad (definiendo estas); si es posible finalmente reducir derecho a deber o deber a derecho; si se deben otorgar derechos especiales a ciertos grupos; si se debe profundizar con esto en temáticas más actuales, etc. Son todos debates éticos, metaéticos y morales muy interesantes, pero no se tratarán ahora en este texto.

\section{5.}

\section{Derechos humanos}

\section{¿Qué son los derechos humanos (DDHH)?}

Los derechos humanos o DDHH (O.N.U., 1948), en sus siglas comunes en español, representan el mayor consenso como sistema moral establecido mundialmente y en la actualidad (s. XXI). Aunque no son un sistema moral totalmente articulado ni tienen una ética evaluativa como tal detrás (aunque suele predominar su justificación mediante el deontologismo kantiano y la noción, a veces ambigua -Diéguez, 2020-, de 'dignidad humana'), suponen una serie de normas recogidas y agrupadas con las que se apela a los diferentes estados modernos a que se comprometan, para alcanzar mayores cuotas de bienestar social. Dispuestos usualmente en formato manual de pocas páginas, como la Declaración se divulgan y se exponen desde diferentes organizaciones mundiales.
Siendo derechos y humanos, puede decirse cómo se ha explicado antes, que prescriben lograr todas aquellas permisiones que cabe conceder a los individuos humanos. Como sistema moral contemporáneo, también asume cierta reciprocidad derecho-deber, y por eso todos los derechos de la Declaración se consideran que imponen deberes, aunque a estos no se los mencione tanto como a los primeros ni se les otorgue tanto énfasis. Por su condición desiderativa y poco sistemática, así por ciertas ambigüedades de las normas presentadas textualmente -unos países $\mathrm{u}$ otros pueden interpretar de diferente manera los DDHH-, como algunas carencias posibles dado el contexto histórico urgente en el que se presentó -los consensos se establecieron sin presentar detenidamente la forma concreta con la que cabría cumplir con los DDHH, y algunas naciones han elaborado sus propios códigos de derechos fundamentales-, es que generalmente han estado también sometidos a cierta polémica y debate filosófico (Ramos Pascua, 2001). Falta también, una sólida indagación que permita dar con maneras óptimas de concienciar sobre la importancia de los DDHH en los países más distantes a éstos. Y preservando la conciencia humanitaria y los DDHH ya alcanzados en las naciones que total o parcialmente se comprometan con ellos de algún modo.

Este último punto cae finalmente dentro de la praxiología como rama filosófica (pero no en el sentido filosófico de la economía austriaca de economía): el estudio teórico y prescriptivo de la acción humana social y sus formas más efectivas de realización. Pero tal tarea no solamente incumbiría a los filósofos, ni a los científicos sin más, también recaería sobre técnicos e ingenieros sociales como juristas, los teóricos de derecho y magistrados. El trabajo grupal transdisciplinar (filósofos-juristas-científicos) y no el individual i. e. aislado, proveerá probablemente de éxitos en esta campaña. Dado que VP > VS, se asume desde la filosofía científica que las normas y leyes morales más sólidas deben supeditar a las leyes convenidas por $X$ nación que puedan entrar en conflicto con ellas, que deberían someterse a las reglas éticas y morales (superiores en prioridad). Aunque de nuevo, el proceder efectivo de tal relación es algo que debe concretarse y atender a varias circunstancias y aspectos variables, sin caer en meros idealismos e ingenuidades. Solo el trabajo desde los ámbitos de la filosofía del derecho y de la jurisprudencia de cada país, pueden especificar las condiciones y el modo en que esto debe hacerse transicionar-refor- 
mar hacia un mayor acercamiento de los DDHH de forma constitucional pero acuciante (ergo también democráticamente). De cualquier forma los DDHH, aun con sus posibles críticas, hoy siguen siendo todo un modelo que cabe seguir aproximadamente, y de conocimiento global.

\subsection{Derechos (y deberes) humanos como normas sobre valores objetivos}

\section{¿Qué son exactamente y cómo deben ser los dere-} chos humanos?

Los DDHH parecen encajar con la ética técnica y la moral efectiva que cabría esperar. Pueden entenderse como el conjunto de todos los derechos y valores VP+VS humanos (los VS y sus normas, al menos compatibles con los VP y sus normas: legítimos). Sin embargo, cabría reformular algunas nociones. Se postulan estas mejoras hipotéticas:

1. Los DDHH asumen unos deberes simétricos, pero, salvo en algunas secciones no se les alude explícitamente, dando alta relevancia a los derechos y libertades individuales. Podría corregirse la Declaración con un listado congruente y reflejo de deberes o responsabilidades humanas, los teóricos RRHH. Ya se han hecho algunos intentos en tal dirección equitativa. Además, cabe alertar que la Declaración no se agota en ella misma y se complementa con otros códigos normativos adicionales. Cabría pues, fijar uno que considerase todos los RRHH simétricamente, y diera lugar a los DDHH\&RRHH o más abreviadamente, los DD\&RRHH.

2. Retomando la distinción moral y axiológica entre VP-VS, también cabría diferenciar en los diferentes artículos de la Declaración, aquellos que tratan acerca de necesidades biopsicosociales, de aquellos que tratan series de deseos o intereses legítimos (la separación de todos los valores básicos objetivos, sobre necesidades, de salud y supervivencia/seguridad, no se requeriría, porque tampoco es muy marcado su trato en los diferentes artículos de la Declaración). Es decir, siendo importantes valores primarios y secundarios, ya se ha dicho que hay conflictos dialécticos entre valores, que éstos dan lugar a los juicios de valor, y que cabe decidir priorizar lo primario a lo se- cundario. Los DDHH pues, afectan a DDHH primarios $y$ DDHH secundarios (DDHHPP y DDHHSS respectivamente si se quiere, o más simple: DDHH1 y DDHH2). Diferenciar los DDHH1 y los DDHH2 es una llave que no sólo puede abrir cerraduras a nivel teorético de rigor, sino también a nivel práctico por cuanto permitiría marcar qué derechos dentro de la Declaración, de chocar entre sí, deben supeditarse a otros y sentar un precedente teórico en su posible aplicación en tribunales de justicia.

3. Si se traslada el sistemismo de la axiología a la ética y lo moral alcanzando a los DDHH de la Declaración, es evidente que se pueden organizar todos los artículos y secciones de forma que se diserten las zonas de bioderechos de las zonas de psicoderechos y socioderechos. Y socioderechos que posiblemente incluirían también a los derechos al menos políticos, económicos, culturales, y ambientales. Esto es algo que está ya parcialmente realizado en el texto original, pero puede acabar de perfilarse con precisión y ajustarse a las críticas de los puntos precedentes. Por lo tanto, en total y considerando los puntos 1., 2. y 3.; 
Tabla 2

Sistematización articulada de derechos y deberes humanos

\begin{tabular}{|l|l|l|l|l|l|l|}
\hline $\begin{array}{c}\text { Derechos y deberes/ } \\
\text { Tipos }\end{array}$ & $\begin{array}{c}\text { Biológicos } \\
\text { (B) }\end{array}$ & $\begin{array}{c}\text { Psicológicos } \\
\text { (S) }\end{array}$ & $\begin{array}{c}\text { Políticos } \\
(\mathbf{T})\end{array}$ & $\begin{array}{c}\text { Económicos } \\
\text { (E) }\end{array}$ & $\begin{array}{c}\text { Culturales } \\
\text { (C) }\end{array}$ & $\begin{array}{c}\text { Ambientales } \\
\text { (A) }\end{array}$ \\
\hline $\begin{array}{l}\text { Derechos primarios } \\
\text { (DDHH1) }\end{array}$ & DDHH1B & DDHH1S & DDHH1T & DDHH1E & DDHH1C & DDHH1A \\
\hline $\begin{array}{l}\text { Deberes primarios } \\
\text { (RRHH1) }\end{array}$ & RRHH1B & RRHH1S & RRHH1T & RRHH1E & RRHH1C & RRHH1A \\
\hline $\begin{array}{l}\text { Derechos secundarios } \\
\text { (DDHH2) }\end{array}$ & DDHH2B & DDHH2S & DDHH2T & DDHH2E & DDHH2C & DDHH2A \\
\hline $\begin{array}{l}\text { Deberes secundarios } \\
\text { (RRHH2) }\end{array}$ & RRHH2B & RRHH2S & RRHH2T & RRHH2E & RRHH2C & RRHH2A \\
\hline
\end{tabular}

6.

\section{Conclusiones}

\section{¿Qué esperar de los valores, normas y derechos tratados desde la filosofía científica?}

En general, un mayor rigor, coherencia y una admitida revisión no-arbitraria y razonable a la que siempre puede estar sometido un sistema ético y moral benefician a cualquier sistema teórico que tenga algún alcance cognitivo, sea teórico o práctico. Además de poder proveer de un mejor sostén para justificar y mejorar los DDHH. Esto es lo que, en global, se plantea desde la filosofía 'científica' en la filosofía práctica; una filosofía práctica clara y científicamente informada (y que ayude a su vez a trabajar en ciencias, tecnologías y técnicas), una filosofía técnica. Todo ello, puesto en movimiento efectivo, debería acercar más la sociedad a las utopías democráticas presentes de civilización y organización política (Quintanilla y Vargas-Machuca, 1989), incluyendo a todo tipo de sujetos de derechos relevantes (Mosterín, 2014), siendo bienvenidos si es de forma justificada.

Cómo síntesis de las conclusiones tentativas ofrecidas desde la filosofía científica en la exposición de una filosofía práctica objetiva i. e. técnica, entonces:

1. Es factible tratar valores objetivos, aún si son ficciones y relativos per se o en sistema.

2. Se pueden erigir sistemas morales provisionales, pero efectivos.

3. Testear o contrastar, de alguna forma las reglas morales, es necesario y viable.

4. Cabe defender razonable, objetiva y empáticamente los DDHH.

5. Ningún sistema moral racional, por válido que sea, es dogmático: cabe actualizar los DD\&RRHH conforme a nuevos conocimientos.

6. Todo valor y sistema de valores, en general, es discutible y provisional a la luz de una filosofía científica. La crítica y la reflexión son siempre bienvenidas con tal de avanzar. 
Ahora cabe pasar a la acción: ensayar sistemas morales, evaluar su eficacia aun si es de forma muy provisoria, y ajustarlos a una teoría ética desarrollada que logre contestar a problemas abiertos, reflexionar sobre estos y generar nuevos problemas fecundos.

\section{Referencias}

Bunge, M. (1989). Treatise on Basic Philosophy, vol. 8: Ethics. Kluwer Academic Publishers. 34-36.

Bunge, M. (2002). Ser, saber, hacer. Editorial Paidós. 33-36, 45-49.

Bunge, M. (2012). Filosofía de la Tecnología y otros ensayos. Fondo Editorial.

Diéguez, A. L. (2012). La opción naturalista; una respuesta a Francisco Soler. Naturaleza y Libertad. Revista de estudios interdisciplinares; $n^{\circ} 1$. 240. https://doi.org/10.24310/nyl.v1i1.3980

Diéguez, A. L. (2020). La apelación a la dignidad en el debate sobre el mejoramiento humano. Cuadernos Hispanoamericanos. https://bit.ly/3ko1Bi6

Ferrater, M. (1999). De la materia a la razón. Alianza Editorial.

Frondizi, R. (1958). ¿Qué son los valores? Introducción a la axiología. Fondo de Cultura Económica.

Haack, S. (2017). Scientism and Its discontents. Rounded Globe. https://bit.ly/3srmoUO

Merton, K. (1972). La ciencia y la estructura social democrática. En F. M. Torner y R. Borques (Eds.), Teoría y Estructura Sociales. (pp. 542552). Fondo de Cultura Democrática.

Moore, G. E. (1922). Principia Ethica, second edition. Cambridge University Press.

Mosterín, J. (2014). El triunfo de la compasión. Alianza Editorial.

Organización de las Naciones Unidas. (1948, diciembre). Declaración Universal de Derechos Hu- manos. https://bit.ly/3bvuViw

Ortega y Gasset, J. (1914). Meditaciones del Quijote. Publicaciones de la Residencia de Estudiantes. 43. "Yo soy yo y mi circunstancia, [...]".

Quintanilla, M. A. y Vargas-Machuca, R. (1989). Utopía racional. Espasa Calpe.

Rawls, J. (2006). Teoría de la justicia, segunda edición en español, sexta reimpresión. Fondo de Cultura Económica.

Ramos Pascua, J. A. (2001). La crítica a la idea de los derechos humanos, en Anuario de derechos humanos. Instituto de Derechos Humanos. 872891. https://dx.doi.org/10.5209/ANDH

Rescher, N. (1999). Razón y valores en la Era científico-tecnológica. Paidós I.C.E. / U.A.B. Pensamiento Contemporáneo $n^{0} 59.87$.

Romero, G. E. (2017). Filosofía científica y los límites de la ciencia. Rev. cient. estud. investig., 6(1). 97-103. https://doi.org/10.26885/rcei.6.1.97

Romero, G. E. (2018). Scientific Philosophy. Springer Editorial. 72. https://doi.org/10.1007/978-3-319-97631-0

Teixidó, O. (2019). Sobre la axiología de G. E. Romero y M. Bunge, versión original. Scientia in verba Magazine, Nullius in Verba Editorial. 4755. https://doi.org/10.5281/zenodo.3842243

Teixidó, O. (2020). Sobre la axiología de G. E. Romero y M. Bunge, versión segunda. https://doi.org/10.5281/zenodo.3953932

Velásquez Giraldo, C. I. (2015). La relación entre filosofía y ciencia en la obra de José Ferrater Mora [Tesis doctoral, Unviersitat Autònoma de Barcelona]. https://bit.ly/3nA3U3c

Zavadivker, N. (2004). Una ética sin fundamentos. Facultad de Filosofía y Letras, Universidad Nacional de Tucumán. 